\title{
PET/MR imaging of inflammatory cardiomyopathy as a two for one deal: Great value or too good to be true?
}

\author{
Zorana Mrsic, MD, ${ }^{\mathrm{a}, \mathrm{b}}$ and Edward A. Hulten, MD, MPH, FACC, FASNC ${ }^{\mathrm{b}, \mathrm{c}}$ \\ a Cardiology Service, Department of Medicine, Walter Reed National Military Medical Center, \\ Bethesda, MD \\ b Uniformed Services University of Health Sciences, Bethesda, MD \\ c Cardiology Service, Department of Medicine, Fort Belvoir Community Hospital, Fort Belvoir, \\ VA
}

Received Jan 23, 2019; accepted Jan 24, 2019

doi: $10.1007 / \mathrm{s} 12350-019-01638-7$

\section{See related article, pp. 2118-2129}

Sarcoidosis is a granulomatous condition of unknown etiology that can affect any organ system. The prevalence of cardiac sarcoidosis (CS), a major cause of morbidity and mortality, is not well known and is estimated around $25 \%$ based on autopsy studies ${ }^{1}$ and up to $55 \%$ based on advanced imaging in patients with biopsy known extra-cardiac sarcoidosis. ${ }^{2}$ Endomyocardial biopsy is insensitive, thus a true gold standard for diagnosis of cardiac CS does not exist. Advanced imaging with 18F-FDG cardiac PET and MRI (CMR) has become crucial in diagnosis, management, and longterm follow-up in patients with suspected and confirmed inflammatory cardiomyopathy, in particular CS. ${ }^{3}$

In CS, cardiac PET and MRI image complementary but distinct pathophysiologic processes. PET, a metabolism-based test, uses radiolabeled 18F-FDG to imageenhanced glucose metabolism in the areas where myocardial inflammation is present. CMR, on the other hand, traditionally uses pre and post-gadolinium-based contrast tissue characterization techniques to image areas of increased interstitial edema and fibrosis or scar. ${ }^{3,4}$ Gadolinium is an extracellular contrast agent, thus areas of increased gadolinium concentration in the

Reprint requests: Edward A. Hulten, MD, MPH, FACC, FASNC, Cardiology Service, Department of Medicine, Fort Belvoir Community Hospital, DeWitt Loop 9300, Fort Belvoir, VA 22060; edward.a.hulten.mil@mail.mil

J Nucl Cardiol 2020;27:2130-4.

$1071-3581 / \$ 34.00$

Copyright (c) 2019 American Society of Nuclear Cardiology. myocardium suggest expansion of the myocardial extracellular volume (ECV) due to inflammation, infiltration, infarction, or fibrosis. CMR does provide diagnostic value regarding active edema/inflammation, but the diagnostic accuracy of CMR-based techniques for inflammation is inferior to PET and less useful clinically. Although both CMR and PET have diagnostic and prognostic implications individually, ${ }^{4-7}$ they have complimentary value in diagnostic accuracy and management of $\mathrm{CS}^{8}{ }^{8}$ Expert centers use either CMR or PET for initial diagnosis of inflammatory cardiac disease such as sarcoidosis, although CMR is favored due to having zero radiation and being highly sensitive. On the other hand, clinicians generally employ PET to clarify difficult diagnostic cases or for serial imaging and to evaluate response to therapy (Figure 1). This concept has sparked interest in hybrid imaging techniques using PET/MRI and development of integrated scanner platforms, which have been rapidly expanding in research and clinical arenas ever since their introduction in 2010. The question has remained if the hybrid PET/MRI platform is able to provide the convenience of a single scan without sacrificing the quality of the individual components.

In this issue of the Journal, Wisenberg et al. prospectively evaluated the imaging characteristics and diagnostic information obtained in a cohort of ten patients with suspected CS who underwent same-day PET/CT and PET/MRI. ${ }^{9}$ They demonstrate similar presence/absence and distribution of 18F-FDG uptake in all subjects, with improved contrast between areas of enhanced myocardial uptake compared to background in patients who underwent PET/MRI. This observation is attributed to greater clearance of tracer from the blood pool and longer scan acquisition times which were used 
Cardiac MRI

Initial Diagnosis

Structure, Function, Tissue Characterization
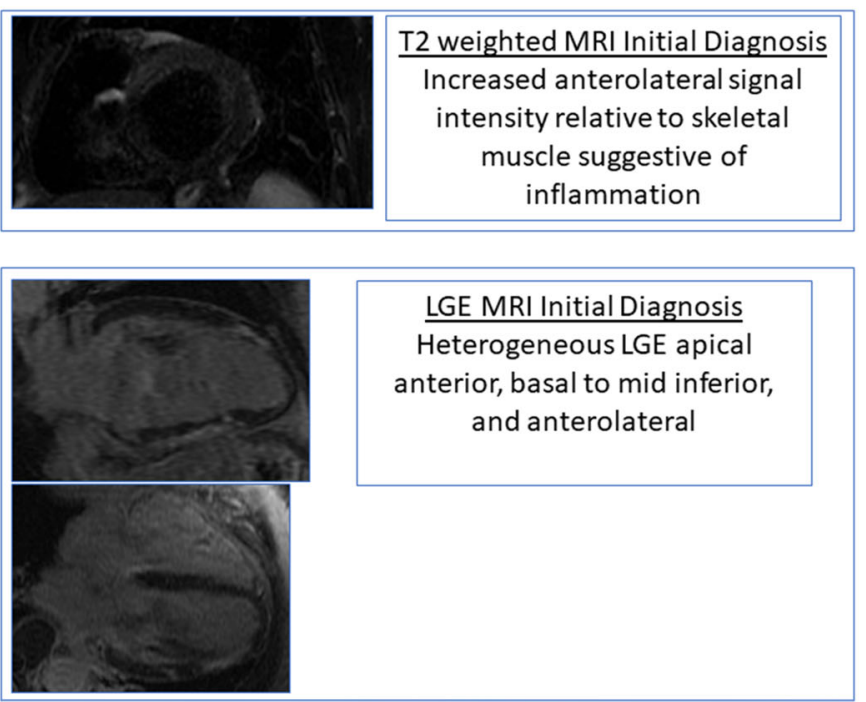

18F-FDG Cardiac PET

Diagnostic clarification of select cases

Serial Follow-up and response to therapy

Macrophage mediated inflammation

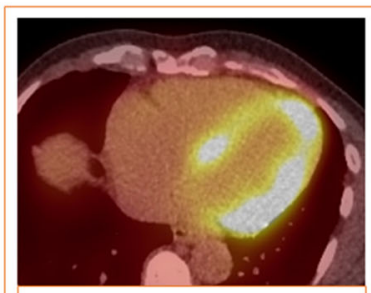

Initial Diagnosis 18F-FDG

Heterogeneous 18F-FDG uptake

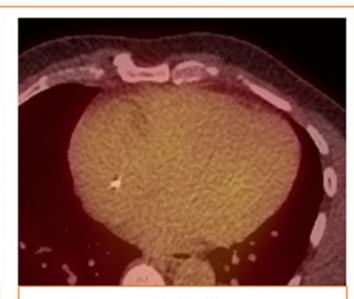

6 month follow up

No 18F-FDG uptake AICD placed

Figure 1. Cardiac MRI and 18F-FDG cardiac PET of a patient with cardiac sarcoidosis. The T2weighted MRI images demonstrated increased myocardial signal intensity relative to skeletal muscle suggestive of inflammation. However, the inflammation demonstrated by 18F-FDG cardiac PET is more accurate and reliable. Late gadolinium enhancement (LGE) images demonstrated heterogeneously focal abnormal signal in a non-coronary distribution indicative of expansion of the myocardial extracellular volume due to fibrosis in this case. Initial 18F-FDG cardiac PET/CT images demonstrated heterogeneous cardiac uptake as well as extra-cardiac uptake consistent with acute inflammation due to active cardiac sarcoidosis that was confirmed by lymph node biopsy. Sixmonth follow-up PET/CT after prednisone therapy demonstrated resolution of abnormal 18F-FDG uptake. AICD, automated internal cardiac defibrillator.

to compensate for the decay given that PET/MRI was performed after the PET/CT. In eight patients who had prior cardiac MRIs, the regions of delayed myocardial enhancement correlate between the dedicated cardiac MRI and the PET/MRI, with the latter providing improved image quality due the use of a $3 \mathrm{~T}$ magnet rather than the $1.5 \mathrm{~T}$ dedicated unit.

Although eloquently designed, the study is limited by all the weaknesses of a small sample size. Given only ten patients, significant heterogeneity of imaging findings exists, and it is difficult to speculate whether these are related to the imaging technique and quality or heterogeneous pathophysiology and disease activity of the subjects. Despite the heterogeneity, the authors are able to demonstrate a significant inverse linear relationship between metabolically active volumes (MAV) in the thorax and left ventricular ejection fraction (LVEF), with an $R^{2}=0.86, P<.01$, which has known implications in management and prognosis of CS. ${ }^{5}$ The generalizability of these findings, however, is limited by the small sample size. Despite the limitations of a small study design, the authors accomplished the goal of demonstrating that combining PET and MRI imaging in evaluation of cardiac sarcoidosis does not result in degradation of imaging quality adding to the growing body of literature supporting this trend (Table 1).

Although the hybrid PET/MRI technique has sparked tremendous interest and enthusiasm, it is important to pause and ask certain questions regarding the benefits, disadvantages, applicability, accessibility, and cost effectiveness of this technology. The current PET/MRI scanners maintain the advantages of the individual techniques by allowing high spatial and temporal resolution, soft tissue characterization, motion correction and soft tissue discrimination, coupled with ability to characterize inflammation. ${ }^{10}$ One of the clear benefits of the hybrid PET/MRI is the ability to decrease the amount of ionizing radiation the patients are exposed to by cutting out the CT scan. Dweck and colleagues reported average radiation exposure of $8.2 \mathrm{mSv}$ in their 
Table 1. Reprinted with permission from Robson et al ${ }^{10}$

\begin{tabular}{|c|c|c|c|c|c|}
\hline & CT & MR & PET & PET/CT & MR/PET \\
\hline \multicolumn{6}{|l|}{ Anatomic imaging } \\
\hline Spatial resolution & Strong & Strong & Weak & Strong & Strong \\
\hline Soft tissue contrast & Weak & Strong & NP & Weak & Strong \\
\hline \multicolumn{6}{|l|}{ Molecular and functional imaging } \\
\hline Molecular imaging & NP & NP & Strong & Strong & Strong \\
\hline Exogenous contrast tissue imaging & Moderate & Strong & NP & Moderate & Strong \\
\hline Tissue characteristics & Weak & Strong & NP & Weak & Strong \\
\hline Temporal resolution & Moderate & Strong & Moderate & Moderate & Strong \\
\hline \multicolumn{6}{|l|}{ Other } \\
\hline Complexity & Strong & Moderate & Moderate & Moderate & Weak \\
\hline Scan time & Strong & Weak & Moderate & Moderate & Weak \\
\hline Cost & Strong & Moderate & Weak & Moderate & Weak \\
\hline Robustness of imaging & Strong & Moderate & Moderate & Moderate & Weak \\
\hline \multicolumn{6}{|l|}{ Potential } \\
\hline Research potential & Moderate & Strong & Weak & Moderate & Strong \\
\hline Translatability & Strong & Moderate & Weak & Strong & Moderate \\
\hline
\end{tabular}

$C T$, computed tomography; $F D G$, fluorodeoxyglucose; $N P$, not possible

PET/MRI patients vs 12.3 in the PET/CT group. ${ }^{11}$ This is especially important in patients who are young and require repeat imaging, such as those with CS. Additionally, in combining the two imaging techniques, the total scan time is decreased from multiple scans on separate days and efficiency and patient comfort are improved.

With these advantages, however, come several disadvantages. The limitations of the individual imaging techniques still remain. The hybrid PET/MRI scan continues to be limited by an inability to study patients with advanced kidney disease due to risk of nephrogenic systemic fibrosis for gadolinium when glomerular filtration rate is below $30 \mathrm{mg} / \mathrm{dL}$. A major drawback to hybrid PET/MRI scanner is the additional challenge presented when imaging via PET/MRI versus PET/CT in the presence of implantable cardiac devices, which are frequently found in patients with CS. Implantable device management of heart block is common and the Heart Rhythm Society recommends considering preventive defibrillator implantation for patients with CS due to high incidence of ventricular arrhythmia and sudden cardiac death. Although MRIconditional pacemaker/ICDs may be safely scanned, they still require pre- and post-scan interrogation for MRI imaging, additional precautions, and contain metal that results in more significant image artifacts on PET/ MRI versus PET/CT. Failed myocardial FDG uptake suppression which is reported anywhere from 30 to
$50 \%,{ }^{11,12}$ in addition to limiting adequate assessment of inflammation, creates a clinical conundrum in the small subset of patients who have increased myocardial FDG uptake but normal MRI imaging. Although less common, early myocardial inflammation detected by PET but not by MRI cannot be fully ruled out in this setting. This drawback is very relevant in patients with CS whose insulin sensitivity maybe altered by steroid treatment. Many operational obstacles further impede the technology. These include the cost of purchasing and operating the integrated scanners, as well as the limited availability of technologists trained in both modalities which have resulted in a limited availability of the PET/ MRI systems, as summarized by Dr. Robson et al. (see Table). ${ }^{10}$

The true generalizability of hybrid PET/MRI for $\mathrm{CS}$, therefore, remains in question. Although the potential benefits are undeniable, the practical value in patients with suspected or confirmed CS is yet to be determined. In patients who have normal cardiac MRI, the addition of PET is unlikely to increase the likelihood of detecting active cardiac sarcoidosis other than for selected cases. This was demonstrated in a study by Vita and colleagues in which only 2 out of 107 subjects were reclassified as having a high probability of CS given abnormal myocardial FDG uptake in the setting of normal MRI. ${ }^{8}$ Additionally, the assessment of CS response to therapy is very robust with PET imaging, ${ }^{5}$ with no clear benefit to longitudinal MRI follow-up. The 


\section{Combine Use of Cardiac MRI and PET for Suspected Cardiac Sarcoidosis}

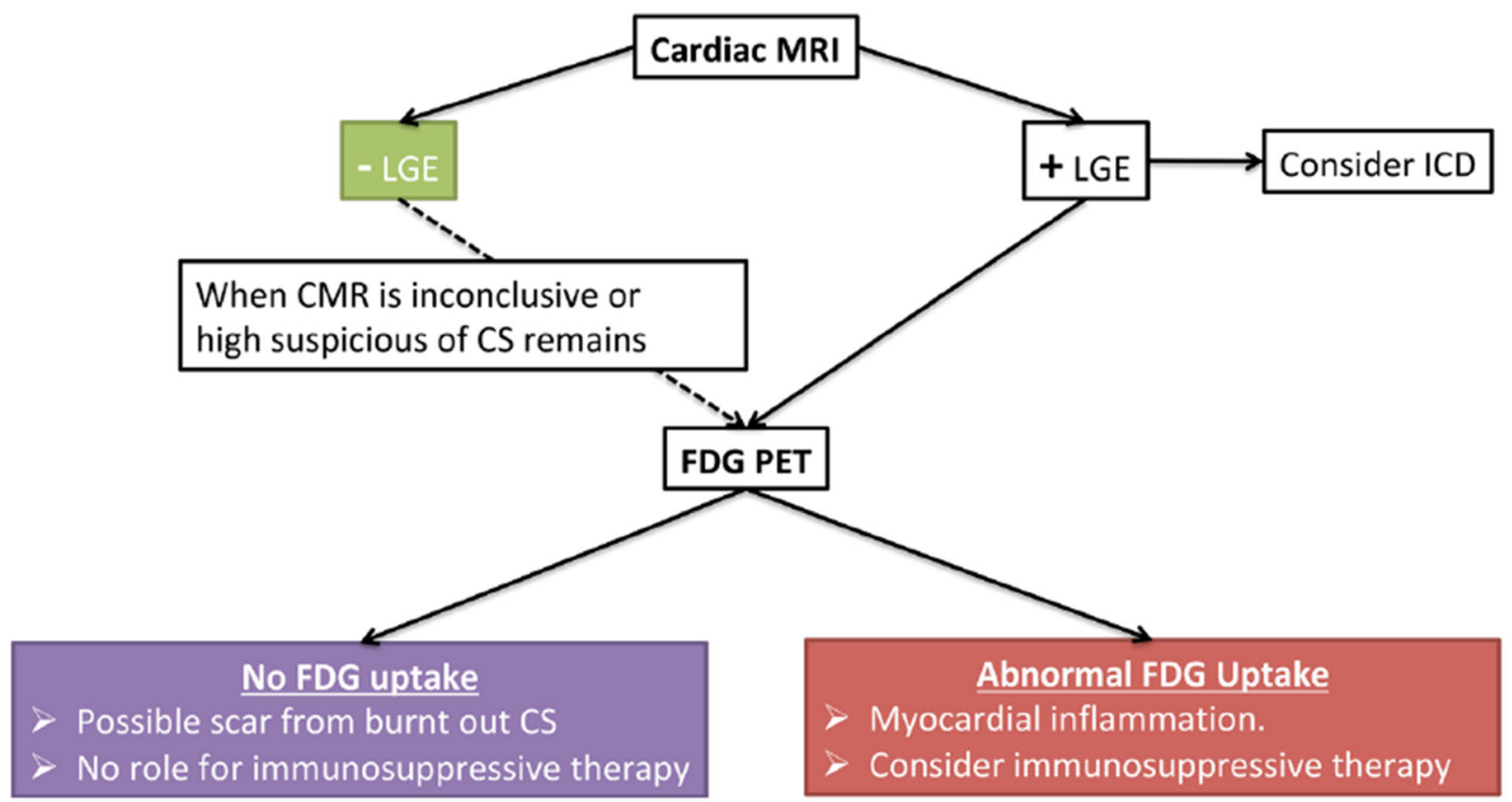

Figure 6. Algorithm for cardiac sarcoidosis (CS) diagnosis. A suggested algorithm for incorporating cardiac magnetic resonance imaging (MRI) and positron emission tomography (PET) for evaluating individuals with suspected CS. CMR indicates cardiac magnetic resonance; FDG, F18-fluorodeoxyglucose; and LGE, late gadolinium enhancement.

Figure 2. Reprinted with permission from Vita et $\mathrm{al}^{8}$.

utility and cost effectiveness of doing simultaneous scans when one can potentially be avoided needs to be further evaluated and a more stepwise imaging approach as suggested by Vita may be more realistic at this time (Figure 2). ${ }^{8}$

It is important to highlight that since the introduction of hybrid PET/MRI scanners, many technological advances have made it more feasible, accurate, and robust and have led to the new applications of this technology. We, therefore, applaud Dr. Wisenberg and colleagues on their contribution in the advancement of this exciting technology. It is only through continued research efforts such as theirs that the previously mentioned challenges can be overcome and the question asked by Dr. Schindler a little over a year ago in this same journal, "Quo Vadis?', answered. ${ }^{13}$

\section{Disclosure}

No financial conflicts of interest. The views expressed in this article are those of the author and do not reflect the official policy of Fort Belvoir Community Hospital, the Defense Health Agency, Department of Defense, or U.S. Government.

\section{References}

1. Iwai K, Takemura T, Kitaici M, Kawabata Y, Matsui Y. Pathological studies on sarcoidosis autopsy. II. Early change, mode of progression and death pattern. Pathol Int 1993;43:377-85.

2. Birnie DH, Sauer WH, Bogun F, Cooper JM, Culver DA, Duvernoy CS, et al. HRS expert consensus statement on the diagnosis and management of arrhythmias associated with cardiac sarcoidosis. Heart Rhythm 2014;11:1304-23.

3. Hulten E, Aslam S, Osborne M, Abbasi S, Bittencourt MS, Blankstein R. Cardiac sarcoidosis: State of the art review. Cardiovasc Diagn Therapy 2016;6:50-63.

4. Hulten E, Agarwal V, Cahill M, Cole G, Vita T, Parrish S, et al. Presence of late gadolinium enhancement by cardiac magnetic resonance among patients with suspected cardiac sarcoidosis is associated with adverse cardiovascular prognosis. Clinical perspective: A systematic review and meta-analysis. Circulation 2016;9:e005001.

5. Osborne MT, Hulten EA, Singh A, Waller AH, Bittencourt MS, Stewart GC, et al. Reduction in 18F-fluorodeoxyglucose uptake on serial cardiac positron emission tomography is associated with improved left ventricular ejection fraction in patients with cardiac sarcoidosis. J Nucl Cardiol 2014;21:166-74.

6. Blankstein R, Osborne M, Naya M, Waller A, Kim CK, Murthy VL, et al. Cardiac positron emission tomography enhances prognostic assessments of patients with suspected cardiac sarcoidosis. J Am Coll Cardiol 2014;63:329-36. 
7. Skali H, Schulman AR, Dorbala S. 18F-FDG PET/CT for the assessment of myocardial sarcoidosis. Curr Cardiol Rep 2013; $15: 352$.

8. Vita T, Okada DR, Veillet-Chowdhury M, Bravo PE, Mullins E, Hulten E, et al. Complementary value of cardiac magnetic resonance imaging and positron emission tomography/computed tomography in the assessment of cardiac sarcoidosis. Clinical perspective. Circulation 2018;11:e007030.

9. Wisenberg G, Thiessen J, Pavlovsky W, Butler J, Wilk B, Prato F. Same day comparison of PET/CT and PET/MR in patients with cardiac sarcoidosis. J Nucl Cardiol 2019. https://doi.org/10.1007/ s12350-018-01578-8.

10. Robson PM, Dey D, Newby DE, Berman D, Li D, Fayad ZA, et al. MR/PET imaging of the cardiovascular system. JACC Cardiovasc Imaging 2017;10:1165-79.
11. Dweck MR, Abgral R, Trivieri MG, Robson PM, Karakatsanis N, Mani $\mathrm{V}$, et al. Hybrid magnetic resonance imaging and positron emission tomography with fluorodeoxyglucose to diagnose active cardiac sarcoidosis. JACC Cardiovasc Imaging 2018;11:94-107.

12. Joshi NV, Vesey AT, Williams MC, Shah AS, Calvert PA, Craighead $\mathrm{FH}$, et al. 18F-fluoride positron emission tomography for identification of ruptured and high-risk coronary atherosclerotic plaques: A prospective clinical trial. Lancet 2014;383:70513.

13. Schindler TH. Cardiovascular PET/MR imaging: Quo vadis? J Nucl Cardiol 2017;24:1007-18.

Publisher's Note Springer Nature remains neutral with regard to jurisdictional claims in published maps and institutional affiliations. 